\title{
Sepsis outcomes in patients receiving statins prior to hospitalization for sepsis: comparison of in-hospital mortality rates between patients who received atorvastatin and those who received simvastatin
}

Daniel R Ouellette ${ }^{1 *}$, Erics Espinoza Moscoso ${ }^{1}$, Julio Pinto Corrales ${ }^{1}$ and Michael Peters ${ }^{2}$

\begin{abstract}
Background: The purpose of this study is to compare the in-hospital mortality rates between septic patients receiving statins and those that did not prior to developing sepsis. We compared subgroups receiving atorvastatin and simvastatin because these two drugs differ in their pharmacologic properties.

Methods: This study was a retrospective analysis of patients selected from an institutional data base of patients hospitalized with sepsis. The study patients were drawn from a data base of 1,961 hospitalized patients with sepsis and included patients who met selection criteria and who were studied for HMG-CoA reductase inhibitor (statin) use both prior to and during hospitalization. The in-hospital mortality rates of patients receiving statins and those that did not prior to developing sepsis were compared. In-hospital mortality rates of patient subgroups receiving atorvastatin and simvastatin were also compared. A multivariable analysis was conducted with in-hospital mortality as the outcome variable and with multiple risk factors to include atorvastatin and simvastatin use.

Results: The mortality rate for 359 patients receiving statins prior to hospitalization for sepsis was not significantly different than that for 1,302 patients who did not receive pre-hospital statins ( $26.5 \%$ versus $30.4 \%, p>0.05)$. The mortality rate for 92 patients who had received atorvastatin prior to hospitalization was significantly less than that of 253 patients who received simvastatin (18.5\% versus 30.0\%, $p=0.032$ ). The use of atorvastatin prior to sepsis was independently associated with lower in-hospital mortality in a multivariable analysis of sepsis risk factors ( $p=0.021$, $\mathrm{OR}=0.455$ ). Patients who received atorvastatin prior to hospitalization for sepsis and had statins continued in hospital had a very low mortality rate that was significantly less than that of those patients who never received statins (15.7\% versus $30.8 \%, p=0.007)$.

Conclusions: Pre-hospital atorvastatin use was associated with improved in-hospital mortality in septic patients when compared with pre-hospital simvastatin use and was independently associated with an improved outcome when compared to other sepsis risk factors. The effect of statins in patients with sepsis may be different for individual statins.
\end{abstract}

Keywords: Sepsis; HMG-CoA reductase inhibitors; Atorvastatin; Critical illness; Mortality; Inflammation

\footnotetext{
*Correspondence: douelle1@hfhs.org

'Pulmonary and Critical Care Medicine, Henry Ford Hospital, K-17, 2799 West Grand Blvd, 48202 Detroit, MI, USA

Full list of author information is available at the end of the article
} 


\section{Background}

Sepsis and septic shock are formidable medical problems that challenge physicians caring for critically ill patients. Today, sepsis is one of the leading causes of morbidity and mortality with thousands of persons suffering from these conditions on a daily basis [1-4]. It has long been recognized that early and aggressive antibiotic treatment reduces mortality from sepsis [5]. Recent advances in resuscitation strategy and methods have also led to improved sepsis outcomes [6]. These advances have been embraced by the medical community and incorporated into guidelines and policy statements [7]. Despite these accomplishments, sepsis mortality remains high, with rates between $20 \%$ and $30 \%$ [8].

Sepsis is characterized by a complex, pleiotropic inflammatory response [8]. Amelioration of the inflammatory cascade in sepsis might be expected to have an impact on the clinical course and outcomes of the patient with sepsis. However, previous efforts to design treatment strategies to modify or disrupt the inflammatory cascade in the septic patient have been largely unsuccessful [9]. Statins are agents which have been observed to have important anti-inflammatory effects and to modulate the immune system response in a variety of ways during sepsis [10]. There has been speculation that the administration of statins may alter the inflammatory response to infection, suggesting that they may represent a potentially important adjunct to therapy [11]. Data suggests that critically ill patients may benefit from statins, and observational and retrospective studies have suggested that patients taking statins prior to the development of sepsis may have improved sepsis outcomes [12-14]. However, a recent large, prospective, multicenter study in patients with acute respiratory distress syndrome (ARDS) due to sepsis failed to demonstrate improved outcomes following the administration of rosuvastatin [15].

Though often considered together as a group, individual statins have unique biological properties. Statins are lipophilic to varying degrees, which may alter their individual effects. In addition, statins have recently been shown to have differential antibacterial properties in vitro, with atorvastatin having more prominent antibacterial effects than other statins [16]. We postulated that individual statins may have an agent-specific effect on outcomes in patients with sepsis and septic shock. We conducted a retrospective review of an institutional data base of patients with severe sepsis. Most patients in our institution who had received statins prior to hospitalization for sepsis had received either atorvastatin or simvastatin. We therefore compared the inpatient mortality rate of patients who had received atorvastatin to those who had received simvastatin.

\section{Methods}

\section{Study design and enrollment}

All patients at our institution who developed sepsis after January, 2005, had medical data entered into an institutional sepsis quality improvement data base. Patients admitted to the intensive care units with a diagnosis of sepsis, as well as those who developed sepsis during the course of their hospital stay for another diagnosis, were included in the data base. Patients with severe sepsis at our institution were transferred to the intensive care units and managed in this venue. We retrospectively reviewed data that was prospectively collected between January 1, 2005, and June 30, 2010. We additionally examined data from the institutional electronic medical record (EMR) and the hospital pharmacy data base for all patients enrolled in the sepsis data base. The study was performed at a quaternary health care system located in the Midwest United States. The study protocol was approved as an exempt protocol by the local institutional review board (IRB) project number 6870. The need for informed consent was waived.

Patient episodes were identified as those patients entered into the sepsis data base where the diagnosis of sepsis was confirmed by a retrospective review of the EMR. If a potential case subject had multiple admissions entered into the data base, only the first admission was considered for study purposes. We reviewed the EMR and the hospital system pharmacy data base to identify if the patient had a record of the use of statins prior to developing sepsis and whether they received statins during their hospitalization for sepsis. We recorded which statin each patient was receiving prior to and during hospitalization. We did not have specific information concerning the pre-hospital duration of treatment with statins. Due to the retrospective nature of the study, we were not able to assess pre-hospital compliance with prescribed statin therapy. The EMR was reviewed to identify the source of sepsis for each patient. We collected data from the EMR for each patient concerning comorbid conditions. The information for each study subject was entered into a research data base without patient identifiers for analysis.

\section{Definitions}

Sepsis was defined as being present if a patient manifested at least two of four systemic inflammatory response syndrome criteria and had documented evidence of infection [17]. Time 0 was defined as the point in time when the sepsis bundle was initiated for each study subject. Intubation was defined as endotracheal intubation and mechanical ventilation within $24 \mathrm{~h}$ of time 0. In-hospital mortality was defined as death from any cause during the hospitalization prior to discharge. Time to antibiotics was defined as the elapsed 
time in minutes from the initial presentation of sepsis to the initial administration of antibiotics.

We performed all statistical analysis using SPSS software version 18 with a logistical regression add-on package version 20 (IBM, Armonk, NY, USA) and considered $p<0.05$ to be statistically significant unless otherwise specified. We used chi-squared tests for univariate analysis of dichotomous variables and two-sample $t$ tests or Mann-Whitney $U$ tests as appropriate for univariate analysis of continuous variables. Adjustments were not made for multiple comparisons. Where mean variables are listed, such data includes the standard deviation (mean \pm standard deviation).

We developed a model to identify risk factors for mortality by multivariable logistic regression analysis in the subject populations. Potential risk factors were identified from a univariable analysis of each of the available variables using mortality as the dependent variable. Variables were selected for analysis if they were significantly associated with mortality $(p<0.05)$ and if at least $80 \%$ of the case and control subjects had data available for the variable.

\section{Results}

Clinical data were collected in a data base of patients with sepsis for 1,965 patient episodes of sepsis between January 1, 2005, and December 31, 2010. From these patient episodes, we selected 1,661 patient episodes of sepsis for study based upon the criteria described in the 'Methods' section (Figure 1). Exclusions included subsequent patient episodes after the first in patients with multiple sepsis events (the majority) and those where demographic data was incomplete. Of these patients, $58 \%$ were transferred to the ICU from the emergency department, $12 \%$ were transferred to the ICU from a general medical or surgical ward, and $29 \%$ were active patients in the ICU when sepsis was diagnosed. Patients received initiation of sepsis care as soon as sepsis was identified, regardless of the venue of care. Of the 1,661 patient episodes investigated, 1,170 resulted in survival to hospital discharge while 491 led to in-hospital death from all causes, providing an overall in-hospital mortality rate of $29.6 \%$ for the investigated population (Figure 1). The mean age ( \pm standard deviation) for the population was $63( \pm 17)$ years, and the mean APACHE II score ( \pm standard deviation) was $19( \pm 7)$. Male patients (53\%) outnumbered female patients in our population. Overall, $44.4 \%$ of patients received vasoactive agents to support blood pressure, and $48.4 \%$ of patients were intubated and received mechanical ventilation within the first $24 \mathrm{~h}$ of sepsis. The primary sources of infection are listed in Table 1.

There were 359 patients who received statins prior to hospitalization for sepsis. The in-hospital mortality rate for patients who received pre-hospital statins was not significantly different from the mortality rate for those patients not receiving pre-hospital statins $(26.5 \%$ versus $30.4 \%, p=0.146$, Figure 2). We chose to compare the group of patients receiving pre-hospital atorvastatin to those receiving simvastatin. The demographic and clinical characteristics of these two groups were very similar (Table 2). Among the patients who received pre-hospital statins, 92 patients received atorvastatin, while 253 received simvastatin (Figure 1). Seventeen patients receiving pre-hospital atorvastatin died (18.5\%), compared with 76 in the simvastatin group (30.0\%), a difference which achieved statistical significance $(p=0.032$, Figure 2$)$. The atorvastatin group also had a significantly lower mortality rate than did those patients not receiving pre-hospital statins $(n=1,302$, mortality rate $=30.4 \%, p=0.015)$. We had dosing information available for all patients receiving pre-hospital simvastatin and atorvastatin. The mortality difference between groups of patients receiving different doses of

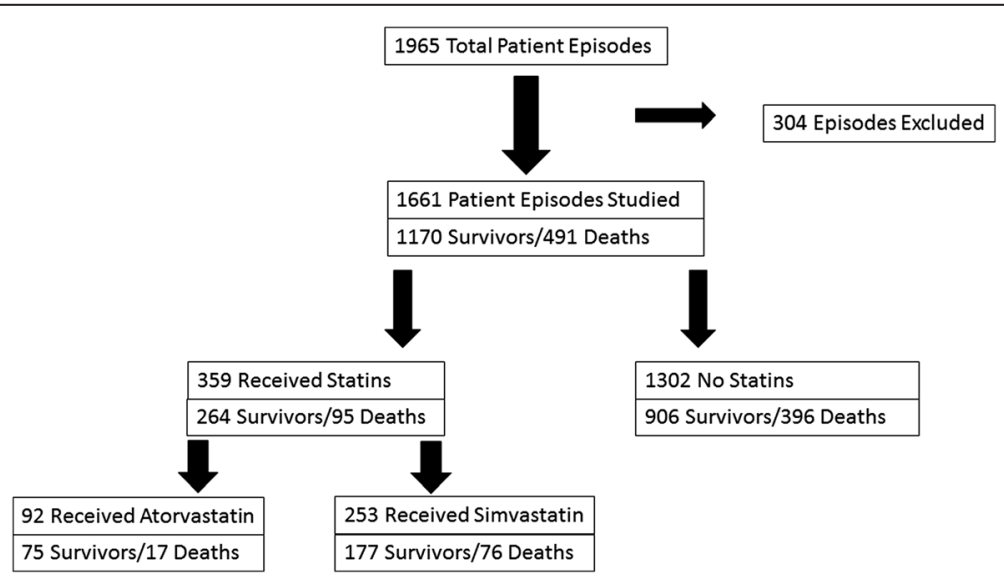

Figure 1 Organizational flowchart of patient episodes categorized by pre-hospital statin use and in-hospital mortality. 
Table 1 Sources of infection

\begin{tabular}{lll}
\hline Source & Number & Percentage of total (\%) \\
\hline Respiratory & 511 & 30.8 \\
Urologic & 263 & 15.8 \\
Abdominal & 217 & 13.1 \\
Skin & 126 & 7.6 \\
Catheter & 51 & 3.1 \\
Blood & 32 & 1.9 \\
Heart & 22 & 1.3 \\
CNS & 19 & 1.1 \\
Bone and joint & 3 & 0.2 \\
Obstetrical & 2 & 0.1 \\
Sinus & 2 & 0.1 \\
Multiple & 110 & 6.6 \\
Unknown & 303 & 18.2
\end{tabular}

simvastatin, or between these groups and the population of patients who did not receive pre-hospital statins, did not achieve significance. For atorvastatin, 5 of the 13 patients prescribed $10 \mathrm{mg}$ of atorvastatin daily died, providing a mortality rate of $38.5 \%$, which compares unfavorably with that of the 79 patients receiving higher doses of atorvastatin $(15.2 \%, p=0.045)$.

In addition to those patients noted who had received atorvastatin or simvastatin, six received lovastatin, five received rosuvastatin, and two received pravastatin. One patient had reported statin use, but the specific agent used was not determined. Two patients died who had received lovastatin, one patient died of the two receiving pravastatin, and no patients receiving rosuvastatin died.

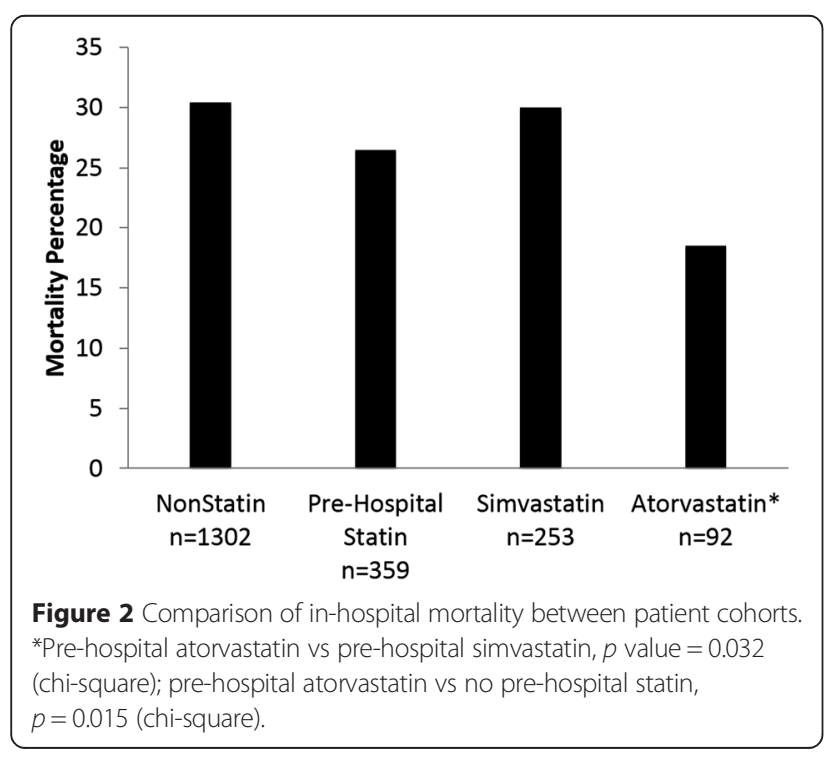

In order to determine if the use of atorvastatin or simvastatin in the pre-hospital setting was an independent risk factor associated with mortality, we performed first a univariable followed by a multivariable analysis of potential risk factors, using mortality as the dependent variable. All 1,661 patient episodes were included in the analysis. We chose a total of 32 potential risk factors to study by univariable analysis, including all the variables listed in Table 2 plus three additional risk factors: prehospital atorvastatin use, pre-hospital simvastatin use, and pre-hospital statin use. Pre-hospital atorvastatin was significantly associated with reduced mortality ( $p=$ $0.018)$, whereas pre-hospital simvastatin was not ( $p=$ $0.856)$ in the univariable analysis. Risk factors that were significantly associated with mortality $(p<0.05)$ in the univariable analysis and for which data was available for at least $80 \%$ of patient episodes were included in the multivariable analysis. The results of the multivariable analysis are presented in Table 3 . We determined that pre-hospital atorvastatin use was significantly and independently associated with improved mortality in septic patients $(p=0.021$, OR $=0.455)$.

Of the 1,302 patients that did not receive pre-hospital statins, 61 patients received statins after hospitalization. The mortality rate of the patients receiving statins in hospital but not before hospitalization was less than that of patients who never received statins, but this difference did not reach significance (23\% versus $30.8 \%$ respectively, $p=0.222$ ). Of the patients who received statins only in hospital, twenty-nine of these patients received atorvastatin with eight such patients expiring (mortality rate $=27.6 \%$ ).

Of the 359 patients who received statins prior to hospitalization, 267 had such therapy continued in hospital. The patients who received statins both prior to hospitalization and during hospitalization had an inhospital mortality rate of $24.7 \%$ compared with a rate of $31.5 \%$ for patients who had received statins prior to hospitalization but had this therapy discontinued (not significant, $p=0.209$ ).

Of the 92 patients who received atorvastatin prior to hospitalization, 70 patients had statins continued in hospital ( 9 patients received simvastatin rather than atorvastatin in-hospital). These 70 patients had a mortality rate of $15.7 \%$, which was significantly different from the population of patients that had never received statins $(p=0.007)$ and was less than but not significantly different from the mortality rate of the 22 patients that had received atorvastatin in the prehospital setting and then who did not receive statins in hospital $(27.2 \%, p=0.223)$. There were 60 patients who received only atorvastatin both prior to and during hospitalization; 9 of these patients died, providing a mortality rate of $15 \%$. 
Table 2 Comparisons between groups defined by pre-hospital statin use

\begin{tabular}{|c|c|c|c|c|c|}
\hline Variable & Atorvastatin & Simvastatin & Significance* & No statins & Significance $^{\#}$ \\
\hline Number of patients & 92 & 253 & & 1,302 & \\
\hline Mean age (years) & $67 \pm 15$ & $69 \pm 13$ & $p=0.365^{a}$ & $61 \pm 17$ & $p=0.001^{\mathrm{a}}$ \\
\hline Gender (male/female) & $40 / 52$ & $130 / 123$ & $p=0.194^{b}$ & $700 / 602$ & $p=0.056^{\mathrm{b}}$ \\
\hline Mean temperature, time $0\left({ }^{\circ} \mathrm{C}\right)$ & $37.3 \pm 1.5$ & $37.2 \pm 1.6$ & $p=0.766^{\mathrm{a}}$ & $37.1 \pm 1.5$ & $p=0.436^{\mathrm{a}}$ \\
\hline Mean heart rate, time 0 , (beats per min) & $106 \pm 22$ & $107 \pm 25$ & $p=0.860^{\mathrm{a}}$ & $112 \pm 25$ & $p=0.033 a$ \\
\hline Mean respiratory rate, time 0 (breaths per min) & $24 \pm 8$ & $24 \pm 9$ & $p=0.512^{\mathrm{a}}$ & $25 \pm 9$ & $p=0.351^{\mathrm{a}}$ \\
\hline Mean arterial pressure, time $0(\mathrm{mmHg})$ & $76 \pm 22$ & $75 \pm 22$ & $p=0.797^{a}$ & $76 \pm 22$ & $p=0.974^{\mathrm{a}}$ \\
\hline Mean arterial $\mathrm{pH}$, time 0 & $7.34 \pm 0.13$ & $7.36 \pm 0.12$ & $p=0.169^{a}$ & $7.35 \pm 0.13$ & $p=0.577^{\mathrm{a}}$ \\
\hline Mean creatinine, time 0 (mg/dL) & $3.5 \pm 3.0$ & $3.1 \pm 2.6$ & $p=0.357^{c}$ & $2.7 \pm 2.9$ & $p=0.003^{c}$ \\
\hline Mean leukocyte count, time $0(\mathrm{~K} / \mu \mathrm{L})$ & $14.7 \pm 9.3$ & $16.5 \pm 12.7$ & $p=0.225^{\mathrm{a}}$ & $16.1 \pm 17.0$ & $p=0.459^{\mathrm{a}}$ \\
\hline Mean hematocrit, time 0 (\%) & $32.6 \pm 6.8$ & $32.3 \pm 6.5$ & $p=0.688^{\mathrm{a}}$ & $32.9 \pm 15.1$ & $p=0.860^{\mathrm{a}}$ \\
\hline Mean platelet count, time $0(\mathrm{~K} / \mu \mathrm{L})$ & $242 \pm 124$ & $238 \pm 130$ & $p=0.813^{\mathrm{a}}$ & $230 \pm 143$ & $p=0.431^{\mathrm{a}}$ \\
\hline Mean bilirubin, time 0 (mg/dL) & $1.2 \pm 1.8$ & $1.1 \pm 2.1$ & $p=0.755^{\mathrm{a}}$ & $1.6 \pm 3.1$ & $p=0.221^{\mathrm{a}}$ \\
\hline Mean lactate (mmol/L) & $3.2 \pm 2.8$ & $3.6 \pm 3.2$ & $p=0.454^{c}$ & $4.0 \pm 3.4$ & $p=0.011^{c}$ \\
\hline Mean APACHE II, time 0 & $18.8 \pm 6.5$ & $19.4 \pm 7.1$ & $p=0.484^{a}$ & $18.6 \pm 7.4$ & $p=0.833^{a}$ \\
\hline Time to antibiotics (min) & $190 \pm 152$ & $166 \pm 155$ & $p=0.300^{\mathrm{a}}$ & $195 \pm 226$ & $p=0.862^{\mathrm{a}}$ \\
\hline Mean fluid volume administered, first $24 \mathrm{~h}(\mathrm{~L})$ & $4.542 \pm 3.166$ & $5.402 \pm 3.348$ & $p=0.036^{\mathrm{a}}$ & $4.955 \pm 4.477$ & $p=0.393^{\mathrm{a}}$ \\
\hline Mean first CVP value $\left(\mathrm{cm} \mathrm{H}_{2} \mathrm{O}\right)$ & $12 \pm 6$ & $11 \pm 6$ & $p=0.361^{\mathrm{a}}$ & $10 \pm 6$ & $p=0.123^{\mathrm{a}}$ \\
\hline Median glucose, first $24 \mathrm{~h}$ (mg/dL) & $142 \pm 82$ & $143 \pm 56$ & $p=0.962^{\mathrm{a}}$ & $134 \pm 67$ & $p=0.226^{\mathrm{a}}$ \\
\hline Mixed venous oxygen saturation (\%) & $71 \pm 12$ & $70 \pm 15$ & $p=0.737^{a}$ & $70 \pm 16$ & $p=0.753^{\mathrm{a}}$ \\
\hline Use of vasopressors, number (\%) & $41(44.6 \%)$ & $115(45.5 \%)$ & $p=0.883^{b}$ & $576(44.2 \%)$ & $p=0.952^{b}$ \\
\hline Intubation, first 24 h, number (\%) & $37(40.2 \%)$ & $118(46.6 \%)$ & $p=0.289^{b}$ & $641(49.2 \%)$ & $p=0.095^{\mathrm{b}}$ \\
\hline Dialysis, number (\%) & $7(7.6 \%)$ & $24(9.5 \%)$ & $p=0.590^{\mathrm{b}}$ & $48(3.7 \%)$ & $p=0.062^{\mathrm{b}}$ \\
\hline Abdominal source, number (\%) & $13(14.1 \%)$ & $29(11.5 \%)$ & $p=0.503^{b}$ & $172(13.2 \%)$ & $p=0.823^{b}$ \\
\hline Lung source (\%) & $27(29.3 \%)$ & $83(32.8 \%)$ & $p=0.542^{\mathrm{b}}$ & $396(30.4 \%)$ & $p=0.314^{b}$ \\
\hline Urologic source (\%) & $16(17.4 \%)$ & $52(20.6 \%)$ & $p=0.514^{b}$ & $192(14.7 \%)$ & $p=0.872^{b}$ \\
\hline History of neoplasia (\%) & $16(17.4 \%)$ & $67(26.5 \%)$ & $p=0.081^{b}$ & $334(25.7 \%)$ & $p=0.077^{\mathrm{b}}$ \\
\hline History of cardiac disease (\%) & $72(78.3 \%)$ & $196(77.4 \%)$ & $p=0.876^{\mathrm{b}}$ & $690(53.0 \%)$ & $p<0.0001^{b}$ \\
\hline History of chronic kidney disease (\%) & $51(55.4 \%)$ & $131(51.8 \%)$ & $p=0.547^{b}$ & $410(31.5 \%)$ & $p<0.0001^{b}$ \\
\hline History of liver disease (\%) & $9(9.8 \%)$ & $20(7.9 \%)$ & $p=0.578^{b}$ & $252(19.4 \%)$ & $p=0.023^{b}$ \\
\hline Mortality (\%) & $17(18.5 \%)$ & $76(30.0 \%)$ & $p=0.032^{b}$ & $396(30.4 \%)$ & $p=0.015^{\mathrm{b}}$ \\
\hline
\end{tabular}

*Comparison between patients receiving atorvastatin or simvastatin prior to hospitalization; ${ }^{*}$ comparison between patients receiving atorvastatin or no statins

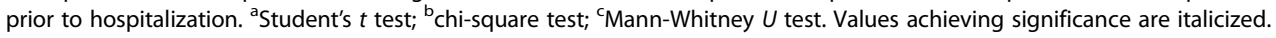

\section{Discussion}

The most important finding in our study is that prehospital atorvastatin use was significantly associated with reduced mortality during a hospitalization for sepsis when compared to pre-hospital simvastatin use. Prehospital administration of atorvastatin was an independent factor associated with improved mortality in our septic population despite the fact that statins as a class were not associated with improved mortality when administered prior to hospitalization. Patients who received atorvastatin prior to a hospitalization for sepsis, and had statins continued in hospital, had a very low mortality rate that was significantly different than patients who never received statins and less than but not significantly different than patients who only received atorvastatin in the pre-hospital setting. Some, but not all, prior studies suggest that administration of statins prior to the development of sepsis may improve sepsis outcomes $[12,18,19]$. Little work has been done in this regard concerning individual statin agents.

Although the focus of our investigations was statin use in septic patients, we also made observations concerning other sepsis mortality risk factors. Risk factors in our patients significantly associated with increased mortality, and those which have been observed by other investigators to be associated with increased mortality during severe infection include thrombocytopenia [20], high 


\begin{tabular}{lll}
\multicolumn{3}{l}{ Table 3 Multivariable analysis of the association of } \\
clinical risk factors with mortality \\
\hline Risk factor & Significance & Odds ratio \\
\hline Platelet count, time 0 & $p=0.001$ & 0.998 \\
Lactate value & $p<0.0001$ & 1.083 \\
APACHE II & $p<0.0001$ & 1.038 \\
Glucose level & $p=0.091$ & 0.998 \\
Male gender & $p=0.073$ & 1.273 \\
Vasopressor use & $p<0.0001$ & 1.678 \\
Mechanical ventilation & $p<0.0001$ & 2.114 \\
Urologic source of infection & $p<0.0001$ & 0.434 \\
Oncologic past history & $p=0.045$ & 1.355 \\
Atorvastatin use prior to sepsis & $p=0.021$ & 0.455 \\
Age & $p=0.003$ & 1.013 \\
Temperature, time 0 & $p=0.091$ & 0.928 \\
Mean arterial pressure, time 0 & $p=0.459$ & 0.998 \\
Leukocyte count, time 0 & $p=0.071$ & 1.010 \\
Hematocrit, time 0 & $p<0.0001$ & 0.956 \\
Arterial pH & $p=0.342$ & 1.796 \\
\hline
\end{tabular}

lactate levels [21], APACHE II score [22,23], vasopressor use $[23,24]$, mechanical ventilation $[23,25]$, a past history of cancer [26], age [23], and anemia [27]. We noted that having a urologic source of sepsis, as opposed to other sources of infection, was associated with a protective effect against mortality, which has also been previously observed [28].

Recent studies have tempered the early enthusiasm for the role of statins in the treatment of sepsis [29]. Janda and coworkers performed a systemic review and meta-analysis of 20 studies, including prospective, retrospective, observational, and cohort studies [19]. A protective effect of statins compared with placebo was demonstrated for a variety of infectious outcomes, but the analysis was very limited by the quality of the studies. Pasin and colleagues assessed five prospective, placebo-controlled studies involving statins in a meta-analysis and found that there was no evidence of a difference in mortality or hospital stay [30]. Wan and associates evaluated both prospective, controlled studies and observational studies [31]. Among five prospective, randomized, controlled studies, there was no demonstrable change in in-hospital or 28-day mortality. However, among 27 observational studies, there was a significant decrease in mortality noted. A recent large, multicenter, prospective, randomized, and controlled study comparing rosuvastatin to placebo in patients with ARDS due to sepsis failed to demonstrate a mortality benefit for rosuvastatin use [15]. In our study, we found that pre-hospital statin use generally did not change outcomes, though atorvastatin was associated with reduced mortality.
Statins are commonly used as lipid-lowering agents. Although often considered as a class, statins vary between agents in terms of their chemical structure and properties and have different pharmacodynamics [32]. While some statins have been derived from fungal metabolites, others, such as atorvastatin, are completely synthetic. Statins vary considerably in terms of their lipophilicity, first-pass metabolism, half-life, and bioavailability. Metabolism of statins varies among agents, and activity at the active site of the enzyme HMGCoA reductase is subtly different for different statins. Because of these chemical and pharmacological differences between statins, different biological effects might be anticipated.

Statins have been postulated to have an effect on sepsis outcomes that is mediated by their anti-inflammatory effects [10,11]. Recent evidence has suggested that statins also may have direct antimicrobial properties. Masadeh and colleagues studied the in vitro antimicrobial effect of statins on 16 common bacterial strains, including both gram-positive and gram-negative bacteria, finding evidence that different statins had varying antimicrobial effects [16]. While both atorvastatin and simvastatin were more potent than rosuvastatin with respect to many gram-positive agents, selected gramnegative organisms were more sensitive to atorvastatin than either simvastatin or rosuvastatin. However, it should be noted that the minimum inhibitory concentrations for atorvastatin, in particular, in the work by Masadeh, exceed by at least 100-fold the maximum serum concentration for this agent seen in human subjects with standard dosing [33]. This fact limits the extrapolation of Masadeh's in vitro data to a clinical effect in sepsis.

Individual statins have not been directly compared with respect to clinical outcomes from infection. We assessed the 27 observational studies highlighted in the comprehensive review by Wan [31], finding that 15 did not distinguish between the types of statins used, and 1 study reported simvastatin use. Of the 11 studies reporting and specifying the individual statins used, none directly compared clinical outcomes between statins. Prospective studies to date have compared individual statins to placebo, but not to other statins. Our work represents the first comparison of in-hospital mortality rates in septic patients treated prior to hospitalization with two different statins, with the finding of improved mortality associated with atorvastatin compared to simvastatin.

Statins have beneficial effects on lipid profiles and cardiovascular outcomes and are generally well tolerated in stable outpatients. Despite the possible beneficial effects noted in this work in patients with sepsis, statins may have adverse effects which could complicate their use, especially in the critically ill patient. Rhabdomyolysis 
and myopathy are known complications of statin use. Statins are metabolized by the cytochrome P450 system in the liver, and liver disease, a common occurrence in the critically ill, affects the metabolism of these agents and increases the risk of muscle disease [32]. Food intake has a variable effect on statin bioavailability, which may have important consequences in the critically ill patient that is unable to receive oral nutrition [32]. Statins are highly protein bound, a fact that may well be important in the critically ill patient [32]. Interruption of chronic outpatient therapy in a critically ill patient could lead to augmentation of cardiovascular risk [14]. We did not have specific information concerning potential adverse effects from statins, including cause-specific mortality and cardiovascular morbidity, which limits our study in recommendations concerning statin utility in sepsis.

Our study has a number of important limitations. Our work is from a single center and is retrospective and observational. We did not have information about prehospital duration of statin therapy, nor did we have information concerning patient compliance with prescribed pre-hospital statin treatments. While most of our patients had health insurance, the lack of coverage for some patients creates the possibility of treatment bias along demographic lines within our population. In addition, insurance policies provide economic advantages for the use of specific agents within a class, a fact which clearly has implications for our study. Given the volatile and changing insurance environment within the United States during the period in question, we have no ability in our retrospective study to completely account for these effects. We performed multiple comparisons in analyzing our data without adjustment, increasing the likelihood of false-positive associations. However, we note that there was little difference between the group of patients receiving atorvastatin and those receiving simvastatin, except for the total volume of fluid received during resuscitation. While resuscitation with intravenous fluid and other agents to defined clinical goals is an important element of sepsis management, we have previously demonstrated in our patients that the total amount of fluid administered in the first $24 \mathrm{~h}$ of the initial resuscitation is not an independent risk factor for adverse clinical outcomes [34].

\section{Conclusions}

Pre-hospital atorvastatin use by patients developing sepsis was associated with reduced in-hospital mortality when compared to the use of pre-hospital simvastatin. Pre-hospital atorvastatin was independently associated with an improved mortality rate when controlling for other sepsis risk factors in a population of septic patients. We postulate that atorvastatin may have unique biochemical, pharmacodynamic, and antimicrobial effects compared with other statins that may account for the observed amelioration in mortality rates. Further study of the effects of individual statin agents in patients with sepsis is needed to confirm this hypothesis.

\section{Abbreviations}

APACHE II: Acute Physiology and Chronic Health Evaluation II; ARDS: Acute respiratory distress syndrome; EMR: Electronic medical record; HMG-CoA: 3-hydroxy-3-methylglutaryl-coenzyme A; IRB: Institutional review board.

\section{Competing interests}

DRO, EEM, and JPC receive grant support for research from Cardeas Pharmaceuticals concerning health care-associated pneumonia in patients receiving mechanical ventilation. All funds go to the institution, and these investigators do not receive salary support from this project. These authors have no other competing interest. MP declares that he has no competing interests.

\section{Authors' contributions}

DRO participated in the design of the study, analyzed and interpreted the data, and helped draft the manuscript. EEM, JPC, and MP participated in the design of the study, were involved in the acquisition of data, and helped draft the manuscript. All authors read and approved the final manuscript.

\section{Acknowledgements}

The authors received no funding for this study.

\section{Author details}

'Pulmonary and Critical Care Medicine, Henry Ford Hospital, K-17, 2799 West Grand Blvd, 48202 Detroit, MI, USA. ${ }^{2}$ Department of Pharmacy, Henry Ford Hospital, K-17, 2799 West Grand Blvd, 48202 Detroit, MI, USA.

Received: 16 December 2014 Accepted: 18 April 2015

Published online: 06 May 2015

\section{References}

1. Angus DC, Linde-Zwirble WT, Lidicker J, Clermont G, Carcillo J, Pinsky MR. Epidemiology of severe sepsis in the United States: analysis of incidence, outcome, and associated costs of care. Crit Care Med. 2001;29:1303-10.

2. Hall JH, Williams SN, DeFrances CJ, Golosinskiy A. Inpatient care for septicemia or sepsis: a challenge for patients and hospitals. NCHS Data Brief 2011; No. 62. Available at: http://www.cdc.gov/nchs/data/databriefs/ db62.htm. Accessed December 2, 2014.

3. Lagu T, Rothberg MB, Shieh M-S, Pekow PS, Steingrub JS, Lindenauer PK. Hospitalizations, costs, and outcomes of severe sepsis in the United States 2003 to 2007. Crit Care Med. 2012;40:754-61.

4. Iwashyna TJ, Ely EW, Smith DM, Langa KM. Long-term cognitive impairment and functional disability among survivors of severe sepsis. JAMA. 2010;304:1787-93.

5. Kumar A, Roberts D, Wood KE, Light B, Parillo JE, Sharma S, et al. Duration of hypotension before initiation of effective antimicrobial therapy is the critical determinant of survival in human septic shock. Crit Care Med. 2006;34:1589-96

6. Rivers E, Nguyen B, Havstad S, REssler J, Muzzin A, Knoblich B, et al. Early goal-directed therapy in the treatment of severe sepsis and septic shock. N Engl J Med. 2001;345:1368-77.

7. Dellinger RP, Levy MM, Rhodes A, Annane D, Gerlach H, Opal SM, et al. Surviving sepsis campaign: international guidelines for management of severe sepsis and septic shock: 2012. Crit Care Med. 2013;41:580-637.

8. Angus DC, van der Poll T. Severe sepsis and septic shock. N Engl J Med. 2013;369:840-51.

9. Angus DC. The search for effective therapy for sepsis: back to the drawing board? JAMA. 2011;306:2614-5.

10. Terblanche M, Almog Y, Rosenson RS, Smith TS, Hackam DG. Statins and sepsis: multiple modifications at multiple levels. Lancet Infect Dis. 2007;7:358-68.

11. Mekontso-Dessap A, Brun-Buisson C. Statins: the next step in adjuvant therapy for sepsis? Intensive Care Med. 2006;32:11-4. 
12. Almog $Y$, Shefer A, Novack V, Maimon N, Barski L, Eizinger M, et al. Prior statin therapy is associated with a decreased rate of severe sepsis. Circulation. 2004;110:880-5.

13. Liappis AP, Kan VL, Rochester CG, Simon GL. The effect of statins on mortality in patients with bacteremia. Clin Infect Dis. 2001;33:1352-7.

14. De Loecker I, Preiser J-C. Statins in the critically ill. Ann Intensive Care. 2012;2:19.

15. The National Heart, Lung, and Blood Institute ARDS Clinical Trials Network. Rosuvastatin for sepsis-associated acute respiratory distress syndrome. N Engl J Med. 2014;370:2191-200.

16. Masadeh M, Mhaidat N, Alzoubi K, Al-azzam S, Alnasser Z. Antibacterial activity of statins: a comparative study of atorvastatin, simvastatin, and rosuvastatin. Ann Clin Microbiol Antimicrob. 2012;11:13.

17. Levy MM, Fink MP, Marshall JC, Abraham E, Angus D, Cook D, et al. SCCM/ ESICM/ACCP/ATS/SIS International Sepsis Definitions Conference. Crit Care Med. 2001;2003(31):1250-6.

18. Kruger P, Bailey M, Bellomo R, Cooper DJ, Harward M, Higgins A, et al. A multicenter randomized trial of atorvastatin therapy in intensive care patients with severe sepsis. Am J Respir Crit Care Med. 2013;187:743-50.

19. Janda S, Young A, FitzGerald JM, Etminan M, Swiston J. The effect of statins on mortality from severe infections and sepsis: a systematic review and meta-analysis. J Crit Care. 2010;25:656.e7-656.e22.

20. Vandijck DM, Blot SI, De Waele JJ, Hoste EA, Vandewoude KH, Decruyenaere JM. Thrombocytopenia and outcome in critically ill patients with bloodstream infection. Heart Lung. 2010;39:21-260.

21. Mikkelsen ME, Miltiades AN, Gaieski DF, Goyal M, Fuchs BD, Shah CV, et al. Serum lactate is associated with mortality in severe sepsis independent of organ failure and shock. Crit Care Med. 2009;37:1670-7.

22. Nguyen HB, Van Ginkel C, Batech M, Banta J, Corbett SW. Comparison of predisposition, insult/infection, response, and organ dysfunction, Acute Physiology and Chronic Health Evaluation II, and mortality in emergency department sepsis in patients meeting criteria for early goal-directed therapy and the severe sepsis resuscitation bundle. J Crit Care. 2012;27:362-9.

23. Mayaud L, Lai PS, Clifford GD, Tarassenko L, Celi LA, Annane D. Dynamic data during hypotensive episode improves mortality predictions among patients with sepsis and hypotension. Crit Care Med. 2013;41:954-62.

24. Povoa PR, Carneiro AH, Ribeiro OS, Pereira AC. Influence of vasopressor agent in septic shock mortality. Results from the Portuguese Community-Acquired Sepsis Study (SACiUCl study). Crit Care Med. 2009;37:410-6.

25. ARISE. ANZICS APD Management Committee. The outcome of patients with sepsis and septic shock presenting to emergency departments in Australia and New Zealand. Crit Care Resusc. 2007;9:8-18.

26. Williams MD, Braun LA, Cooper LM, Johnston J, Weiss RV, Qualy RL, et al. Hospitalized cancer patients with severe sepsis: analysis of incidence, mortality, and associated costs of care. Crit Care. 2004;8:R291-8.

27. Reade MC, Weissfeld L, Angus DC, Kellum JA, Milbrandt EB. The prevalence of anemia and its association with 90-day mortality in hospitalized community acquired pneumonia. BMC Pulm Med. 2010;10:15.

28. Levy M, Dellinger $P$, Townsend SR, Linde-Zwirble WT, Marshall JC, Bion $J$, et al. The surviving sepsis campaign: results of an international guideline-based performance improvement program targeting severe sepsis. Crit Care Med. 2010;38:367-74.

29. Leung S, Pokharel R, Gong MN. Statins and outcomes in patients with blood stream infection: a propensity-matched analysis. Crit Care Med. 2012;40:1064-71.

30. Pasin L, Landoni G, Castro ML, Cabrini L, Belletti A, Feltracco P, et al. The effect of statins on mortality in septic patients: a meta-analysis of randomized controlled trials. PLoS One. 2013:8:e82775.

31. Wan Y-D, Sun T-W, Kan Q-C, Guan F-X, Zhang S-G. Effect of statin therapy on mortality from infection and sepsis: a meta-analysis of randomized and observational studies. Crit Care. 2014;18:R71.

32. Schachter M. Chemical, pharmacokinetic and pharmacodynamic properties of statins: an update. Fundam Clin Pharmacol. 2005;19:117-25. doi:10.1111/ j.1472-8206.2004.00299.x.

33. Stern RH, Bing-Bing Y, Hounslow NJ, MacMahon M, Abel RB, Olson SC. Pharmacodynamics and pharmacokinetic-pharmacodynamic relationships of atorvastatin, an HMG-CoA reductase inhibitor. J Clin Pharmacol. 2000;40:616-23.

34. Ouellette DR, Shah SZ. Comparison of outcomes from sepsis between patients with and without pre-existing left ventricular dysfunction: a case-control analysis. Crit Care. 2014;18:R79.

\section{Submit your manuscript to a SpringerOpen ${ }^{\circ}$ journal and benefit from:}

- Convenient online submission

- Rigorous peer review

- Immediate publication on acceptance

- Open access: articles freely available online

- High visibility within the field

- Retaining the copyright to your article

Submit your next manuscript at $>$ springeropen.com 